\title{
Two-connected networks with rings of bounded cardinality
}

\author{
B. Fortz $\quad$ M. Labbé ${ }^{\dagger}$
}

June 12, 2002

\begin{abstract}
We study the problem of designing at minimum cost a two-connected network such that each edge belongs to a cycle using at most $K$ edges. This problem is a particular case of the two-connected networks with bounded meshes problem studied by Fortz, Labbé and Maffioli [6].

In this paper, we compute a lower bound on the number of edges in a feasible solution, we show that the problem is strongly NP-complete for any fixed $K$, and we derive new classes of facet defining inequalities. Numerical results obtained with a branch-and-cut algorithm using these inequalities show their effectiveness for solving the problem.
\end{abstract}

Keywords: network design, combinatorial optimization, branch-and-cut

\section{Introduction}

Telecommunication network planning has become in the last decade an important problem area for developing and applying optimization models. Telephone companies have initiated extensive modeling and planning efforts to expand and upgrade their transmission facilities.

Recently, Fortz et al. [6] introduced a new model for the topological design of backbone telecommunication networks. The two-connected network with bounded rings (or meshes) problem (2CNBR) consists in designing a minimum cost network $N$ with the following constraints:

1. $N$ contains at least two node-disjoint paths between every pair of nodes (2-connectivity constraints),

and

2. each edge of $N$ must belong to at least one cycle whose length is bounded by a given constant $K$ (ring constraints).

\footnotetext{
${ }^{*}$ Institut d'Administration et de Gestion, Université Catholique de Louvain, Place des Doyens 1, B-1348 Louvainla-Neuve, Belgium (fortz epoms.ucl.ac.be)

†Institut de Statistique et de Recherche Opérationnelle, SMG, CP 210/01, Univ. Libre de Bruxelles, Bd du Triomphe, B-1050 Bruxelles, Belgium (mlabbeesmg.ulb.ac.be)
} 
In the most common mathematical model for topological network design, a graph $G=(V, E)$ is considered where $V$ is the set of nodes that have to be connected and $E$ is the set of edges, that is the set of potential links between nodes. Each edge $e$ of $E$ has a fixed nonnegative $\operatorname{cost} c_{e}$ and the objective is to find the subset $F$ of $E$ of minimum total cost, such that the resulting network $N=(V, F)$ satisfies some survivability requirement. This requirement can be that the network be either $k$-edge-connected or $k$-node-connected, which means that the removal of any $(k-1)$ or fewer edges (respectively, nodes) leaves $G$ connected.

In most cases, two-connected networks have been found to provide a sufficient level of survivability. Hence, a considerable amount of research has focused on so-called low-connectivity constrained network design problems, i.e. problems for which each node $j$ is characterized by a requirements $r_{j} \in\{0,1,2\}$ and $\min \left\{r_{v}, r_{w}\right\}$ node-disjoint paths between every pair of distinct nodes $v, w$ are required. Work on this kind of problem goes from the early contributions of Steiglitz et al. [12] to the more recent articles of Grötschel and Monma [7], Boyd and Hao [3], Monma and Shallcross [10], Grötschel et al. [8,9], and others. For in depth surveys in this area the reader is referred to Fortz [4] and Grötschel et al. [9].

The minimum-cost two-connected network is often a Hamiltonian cycle. Therefore, any edge failure would require to reroute the flow that passed through that edge, using all the edges of the network, an obviously undesirable feature. Fortz et al. [6] proposed to add ring constraints to limit the region of influence of the traffic which is necessary to reroute if a connection is broken. They implemented a first branch-and-cut algorithm to solve the problem as well as several constructive heuristics. More recently, Fortz and Labbé [5] studied the structure of the underlying polyhedra, deriving new classes of facet-defining inequalities.

An important application of ring constraints appear in topologies using the recent technology of self-healing rings. Self-healing rings are cycles in the network equipped in such a way that any link failure in the ring is automatically detected and the traffic rerouted by the alternative path in the cycle. Due to technological constraints, the length of self-healing rings must be limited. This is equivalent to set a bound on the length of the shortest cycle including each edge. In practice, the length of the ring is computed as the number of hops, i.e. the number of nodes that compose the ring. This corresponds to the particular case of $2 \mathrm{CNBR}$ that arises when a unit length is given to each edge.

In this paper, we derive additional properties for this particular case. The next section introduces some notation and a mathematical formulation for our model. In Section 3, we compute a lower bound on the number of edges in any feasible solution. This bound is used in Section 4 to show that the problem is NP-complete even for $K$ fixed, and is then extended to a new class of valid inequalities in Section 5. The separation problem for these inequalities is studied in Section 6, and a branch-and-cut algorithm is outlined in Section 7. Numerical results with this algorithm are presented in Section 8.

\section{Notation and model}

As mentioned before, we represent the given set of nodes and possible cable connections by an undirected graph $G=(V, E)$. Throughout this paper, $n:=|V|$ and $m:=|E|$ will denote the number of nodes and edges of $G$. 
Given the graph $G=(V, E)$ and $W \subset V$, the edge set

$$
\delta(W):=\{\{i, j\} \in E \mid i \in W, j \in V \backslash W\}
$$

is called the cut induced by $W$. We write $\delta_{G}(W)$ to make clear — in case of possible ambiguities with respect to which graph the cut induced by $W$ is considered. The degree of a node $v$ is the cardinality of $\delta(v)$. The set

$$
E(W):=\{\{i, j\} \in E \mid i \in W, j \in W\}
$$

is the set of edges having both end nodes in $W$. We denote by $G(W)=(W, E(W))$ the subgraph induced by edges having both end nodes in $W$. If $E(W)$ is empty, $W$ is an independent set. $G / W$ is the graph obtained from $G$ by contracting the nodes in $W$ to a new node $w$ (retaining parallel edges).

We denote by $V-z:=V \backslash\{z\}$ and $E-e:=E \backslash\{e\}$ the subsets obtained by removing one node or one edge from the set of nodes or edges, and $G-z$ denotes the graph $(V-z, E \backslash \delta(\{z\}))$, i.e. the graph obtained by removing a node $z$ and its incident edges from $G$. This is extended to a subset $Z \subset V$ of nodes by the notation $G-Z:=(V \backslash Z, E \backslash(\delta(Z) \cup E(Z)))$.

Each edge $e:=\{i, j\} \in E$, has a fixed $\operatorname{cost} c_{e}:=c_{i j}$ representing the cost of establishing the direct link connection. The cost of a network $N=(V, F)$ where $F \subseteq E$ is a subset of possible edges is denoted by $c(F):=\sum_{e \in F} c_{e}$. The distance between two nodes $i$ and $j$ in this network is denoted by $d_{F}(i, j)$ and is given by the minimum number of edges in a path linking these two nodes in $F$.

A useful tool to analyze feasible solutions of $2 \mathrm{CNBR}$ is the restriction of a graph to bounded rings. Given a graph $G=(V, E)$ and a constant $K>0$, we define for each subset of edges $F \subseteq E$ its restriction to bounded rings $F_{K}$ as

$$
F_{K}:=\left\{e \in F: \begin{array}{l}
e \text { belongs to at least one cycle } \\
\text { of length less than or equal to } K \text { in } F
\end{array}\right\} .
$$

The subgraph $G_{K}=\left(V, E_{K}\right)$ is the restriction of $G$ to bounded rings. Remark that an edge $e \in E \backslash E_{K}$ will never belong to a feasible solution of $2 \mathrm{CNBR}$.

In order to formulate the $2 \mathrm{CNBR}$ problem, we associate with every subset $F \subseteq E$ an incidence vector $\mathbf{x}^{F}=\left(x_{e}^{F}\right)_{e \in E} \in\{0,1\}^{|E|}$ by setting

$$
x_{e}^{F}:= \begin{cases}1 & \text { if } e \in F \\ 0 & \text { otherwise } .\end{cases}
$$

Conversely, each vector $\mathbf{x} \in\{0,1\}^{|E|}$ induces a subset

$$
F^{\mathbf{x}}:=\left\{e \in E \mid x_{e}=1\right\}
$$

Further we denote by $\mathcal{D}_{G, K}$ the set of incidence vectors $x^{F}$ with $F \subseteq E$ such that

1. $F$ is two-connected,

2. $F=F_{K}$. 
Then, the 2CNBR problem consists in

$$
\min \left\{\sum_{e \in E} c_{e} x_{e}: x \in \mathcal{D}_{G, K}\right\} .
$$

Since all costs $c_{e}, e \in E$ are assumed to be positive, there always exists an optimal solution of $2 \mathrm{CNBR}$ whose induced graph is minimal with respect to inclusion. Hence, 2CNBR can be equivalently formulated as

$$
\min \left\{\sum_{e \in E} c_{e} x_{e}: x \in \mathbb{Z}^{+} \text {and there exists } y \in \mathcal{D}_{G, K}: y \leq x\right\} .
$$

For any subset of edges $F \subseteq E$ we define

$$
x(F):=\sum_{e \in F} x_{e}
$$

We also denote by $e_{i}$ the $i$-th unit vector in $\mathbb{R}^{n}$.

If a subset of edges $S \subseteq E$ is such that $(G-S)_{K}$ is not two-connected, then $G-S$ does not contain a feasible solution, and therefore each feasible solution contains at least one edge from $S$. As we are only interested in minimal feasible solutions, this is sufficient to formulate the $2 \mathrm{CNBR}$ problem as the following integer linear program :

$$
\begin{array}{cll}
\min & \sum_{e \in E} c_{e} x_{e} & \\
\text { s.t. } & & \\
& x(S) \geq 1 & S \subseteq E,(G-S)_{K} \text { is not two-connected, } \\
& x_{e} \geq 0 & e \in E, \\
x_{e} \text { integer } & e \in E .
\end{array}
$$

Constraints (1) are called subset constraints and provide a set covering formulation of the 2CNBR problem. This formulation was introduced by Fortz and Labbé [5]. They also characterized which subset constraints are facet-defining for

$$
\mathcal{P}_{G, K}:=\operatorname{conv}\left\{x \in \mathbb{R}^{|E|}: x \text { satisfies }(1)-(3)\right\}
$$

the polyhedron associated to the $2 \mathrm{CNBR}$ problem. This polyhedron is fully dimensional.

\section{A lower bound on the number of edges}

In this section, we compute a lower bound on the number of edges in any feasible solution of 2CNBR. This result is useful for showing that the problem is NP-complete for any fixed $K \geq 3$ and for deriving new valid inequalities.

We first need the following definitions and properties from graph theory. Let $G=(V, E)$ be a connected graph with $n=|V|$ nodes and $m=|E|$ edges, and let $T$ be a spanning tree in $G$. 


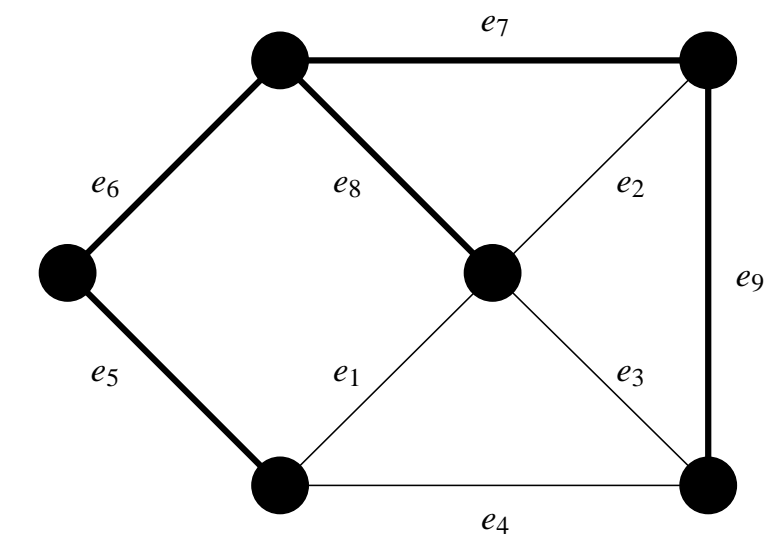

$$
C=\left(\begin{array}{cccc|ccccc}
e_{1} & e_{2} & e_{3} & e_{4} & e_{5} & e_{6} & e_{7} & e_{8} & e_{9} \\
\hline 1 & 0 & 0 & 0 & 1 & 1 & 0 & 1 & 0 \\
0 & 1 & 0 & 0 & 0 & 0 & 1 & 1 & 0 \\
0 & 0 & 1 & 0 & 1 & 1 & 1 & 0 & 1 \\
0 & 0 & 0 & 1 & 1 & 1 & 1 & 0 & 1
\end{array}\right)
$$

Figure 1: An example of graph and its fundamental cycle matrix

The addition of an edge to $T$ creates exactly one cycle, called a fundamental cycle. Since there are $m-n+1$ chords in $G$, there are exactly $m-n+1$ fundamental cycles associated with each spanning tree.

Now, suppose the edges of $G$ are ordered as $e_{1}, \ldots, e_{m}$, and we have a set of cycles $\sigma_{i}, i=$ $1, \ldots, k$. Then, we can define a cycle matrix $C=\left(c_{i j}\right)_{k \times m}$ in which

$$
c_{i j}= \begin{cases}1 & \text { if } \sigma_{i} \text { contains edge } e_{j} \\ 0 & \text { otherwise }\end{cases}
$$

If the cycles $\sigma_{i}$ are the fundamental cycles associated to a spanning tree $T$, this matrix is called fundamental cycle matrix. Moreover, if edges in $G$ are numbered starting from the edges not belonging to $T$, and the fundamental cycles are numbered accordingly, then the fundamental cycle matrix has the following form :

$$
C=\left(I_{\mu} ; C_{t}\right),
$$

where $I_{\mu}$ is an identity matrix of dimension $\mu=m-n+1$, and $C_{t}$ is the remaining matrix of dimension $\mu \times(n-1)$ corresponding to the edges of $T$.

Figure 1 shows an example of graph with its associated fundamental cycle matrix, where the fundamental cycles are defined by the spanning tree drawn in bold.

It is clear that the rank of the fundamental cycle matrix $C$ is $\mu=m-n+1$. With respect to scalars in $\{0,1\}$ and the addition modulo 2 (that we denote by $\oplus$ ), the fundamental cycles are independent, and we can define a vector space for which the fundamental cycles form a basis. This vector space is called the cycle space, and all the other cycles in $G$ can be obtained as a linear combination of rows representing the fundamental cycles in $C$. The dimension $\mu$ of the cycle space is also called the cyclomatic number of the graph.

More details about fundamental cycles and the cycle space can be found e.g. in Berge [2]. 
We can now prove the first important result of this section.

Theorem 1 Let $G=(V, E)$ be a two-edge-connected network with $n=|V|$ nodes and $m=|E|$ edges. If there exists a covering of the edges of the network by cycles containing at most $K$ nodes each, then there exists such a covering using at most $\mu=m-n+1$ independent cycles.

Proof. Since $G$ is two-edge-connected, each edge belongs to a cycle, which can be obtained by a linear combination of the elements of a basis of the cycle space. This means that each edge must belong to at least one cycle in a basis, or, in other words, that a basis of the cycle space covers the edges of the network. Moreover, each cycle matrix of rank $\mu$ defines a basis of the cycle space. This implies that the set of cycles defined by any cycle matrix of rank $\mu-$ and in particular, fundamental cycles - covers the edges of the network.

Let $C$ be a fundamental cycle matrix for $G$. If a subset of the fundamental cycles that use at most $K$ nodes covers the edges, it forms the requested covering. Otherwise, we transform $C$ in a cycle matrix $C^{\prime}$ of rank $\mu$ such that the subset of cycles of $C^{\prime}$ that use at most $K$ nodes covers the network.

In order to obtain that transformation, we now show that a cycle matrix of rank $\mu$, defining $r(r \leq \mu)$ cycles using at most $K$ nodes, and such that these $r$ cycles do not cover the set of edges $E$, can be transformed into a cycle matrix of rank $\mu$, defining $r+1$ cycles using at most $K$ nodes. By applying this construction iteratively to the fundamental cycle matrix $C$, we create a sequence of matrices of rank $\mu$, thus covering the edges of the network by independent cycles, and such that the number of cycles using at most $K$ nodes is increased by one at each step. We stop when these cycles using at most $K$ nodes cover the network, i.e. these form the requested covering. This occurs in a finite number of steps, since in the worst case, we end with a matrix containing $\mu$ cycles, each using at most $K$ nodes, and that cover all the edges.

Let $C_{1}$ be a $\mu \times m$ cycle matrix of rank $\mu$ and suppose there are $r$ cycles using at most $K$ nodes in $C_{1}$. Without loss of generality, we can suppose these cycles form the $r$ first rows of $C_{1}$, i.e. $C_{1}$ is of the form

$$
C_{1}=\left(\begin{array}{c}
C_{r} \\
C_{t}
\end{array}\right),
$$

where $C_{r}$ is a $r \times m$ cycle matrix such that each cycle defined by $C_{r}$ uses at most $K$ nodes and $C_{t}$ is a $(\mu-r) \times m$ cycle matrix such that each cycle defined by $C_{t}$ uses at least $K+1$ nodes.

Suppose cycles in $C_{r}$ do not cover the network, i.e. there exists an edge $e \in E$ which does not belong to any cycle in $C_{r}$. This edge must belong to a cycle $\sigma$ using at most $K$ nodes. Since $C_{1}$ is of rank $\mu, \sigma$ can be obtained by a linear combination of its rows. Moreover, since no cycle in $C_{r}$ uses $e$, the linear combination uses at least one row of $C_{t}$. Replacing this row of $C_{t}$ by the linear combination of rows defining $\sigma$, we obtain a new cycle matrix $C_{2}$ of same rank $\mu$ and defining $r+1$ cycles using at most $K$ nodes.

Interestingly, Theorem 1 still holds if the graph $G$ contains multiple edges. Note that the cycles in the cover do not necessarily correspond to a spanning tree. The constructive proof of Theorem 1 is illustrated by the following example. Consider the graph of Figure 2 with the fundamental cycles associated to the spanning tree drawn in bold, and suppose the rings must use at most 3 nodes. The 


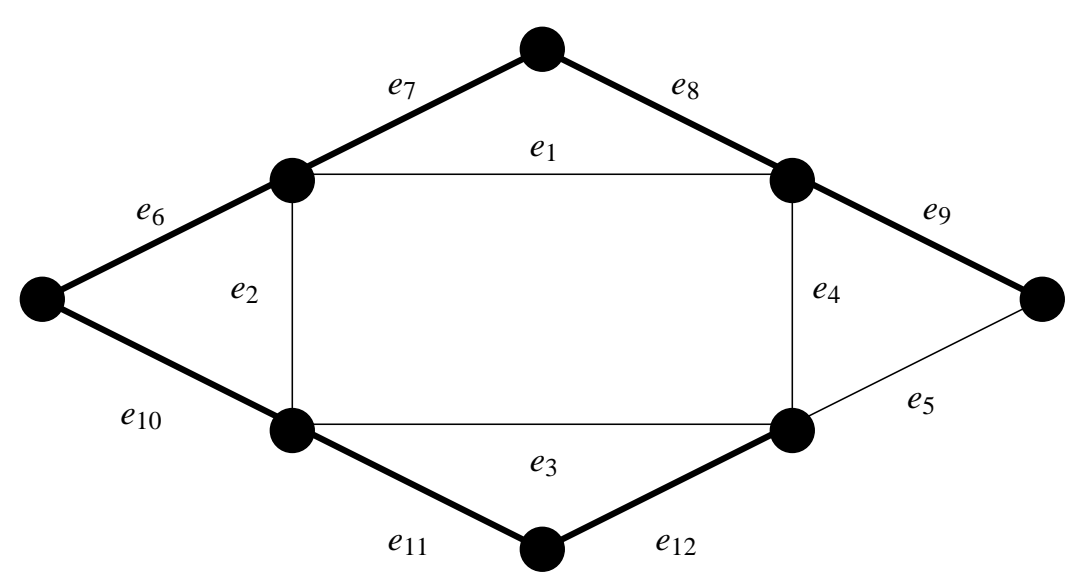

Figure 2: An illustration of Theorem 1

fundamental cycle matrix is

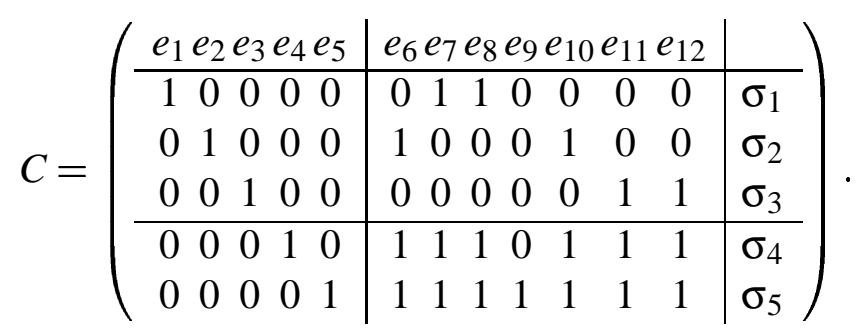

The matrix $C_{r}$ of cycles using at most 3 nodes consists of the first 3 rows of $C$. However, the column corresponding to $e_{4}$ in $C_{r}$ is null, meaning $e_{4}$ is not covered by $C_{r}$. But $e_{4} \in \bar{\sigma}=\left\{e_{4}, e_{5}, e_{9}\right\}$ and $\bar{\sigma}$ is obtained by combining $\sigma_{4}$ and $\sigma_{5}$. We can thus replace $\sigma_{4}$ by $\bar{\sigma}$ and we obtain the new matrix

$$
C^{\prime}=\left(\begin{array}{ccccc|ccccccc|c}
e_{1} & e_{2} & e_{3} & e_{4} & e_{5} & e_{6} & e_{7} & e_{8} & e_{9} & e_{10} & e_{11} & e_{12} & \\
\hline 1 & 0 & 0 & 0 & 0 & 0 & 1 & 1 & 0 & 0 & 0 & 0 & \sigma_{1} \\
0 & 1 & 0 & 0 & 0 & 1 & 0 & 0 & 0 & 1 & 0 & 0 & \sigma_{2} \\
0 & 0 & 1 & 0 & 0 & 0 & 0 & 0 & 0 & 0 & 1 & 1 & \sigma_{3} \\
0 & 0 & 0 & 1 & 1 & 0 & 0 & 0 & 1 & 0 & 0 & 0 & \bar{\sigma} \\
\hline 0 & 0 & 0 & 0 & 1 & 1 & 1 & 1 & 1 & 1 & 1 & 1 & \sigma_{5}
\end{array}\right)
$$

Cycles using at most 3 nodes are given by the first 4 rows, and no column of the corresponding submatrix is null, meaning $\sigma_{1}, \sigma_{2}, \sigma_{3}$ and $\bar{\sigma}$ form a covering of the network as requested in Theorem 1 .

Theorem 1 is the key to establish the main result of this section.

Theorem 2 Let $G=(V, E)$ be a two-connected network with $n=|V|$ nodes and $m=|E|$ edges, such that there exists a covering of the network by cycles using at most $K$ nodes. Then,

$$
m \geq M(n, K):=n+\min \left(\left\lceil\frac{n-K}{K-2}\right\rceil,\left\lceil\frac{n}{K-1}\right\rceil\right),
$$

i.e. $G$ contains at least $M(n, K)$ edges.

Proof. From Theorem 1, there exists a covering of $G$ by at most $m-n+1$ independent cycles using at most $K$ nodes. We consider two disjoint cases : 


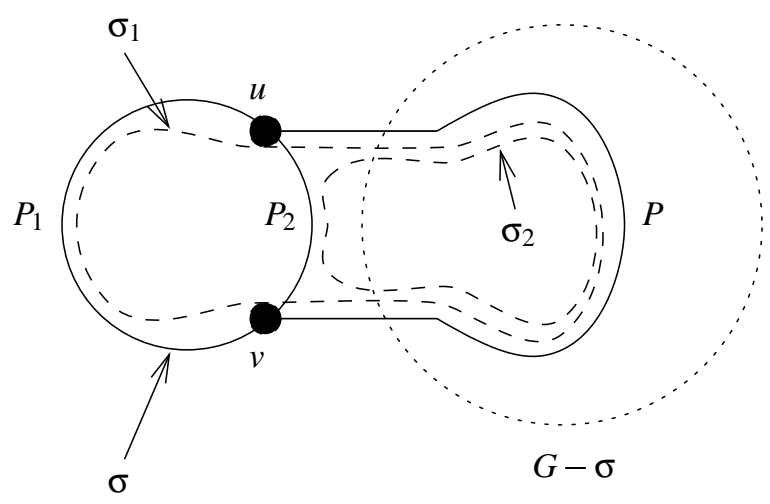

Figure 3: Combinations of a cycle $\sigma$ and an outside path $P$ to generate cycles $\sigma_{1}$ and $\sigma_{2}$

1. There exists a covering satisfying Theorem 1 and using at most $m-n$ cycles.

In this case, the sum of the number of edges used in each cycle is greater than or equal to $m$, since the cycles cover the network. Moreover, since each cycle uses at most $K$ edges, we have

$$
m \leq(m-n) K,
$$

and the integrality of $m$ allows to conclude that

$$
m \geq n+\left\lceil\frac{n}{K-1}\right\rceil \text {. }
$$

2. All coverings satisfying Theorem 1 use exactly $m-n+1$ cycles.

It means that there exists such a covering which is also a basis of the cycle space. We first show that each cycle in this covering shares at least one edge with the others. Suppose it is not the case for some cycle $\sigma$ in the covering. Since the network is two-connected, there exist two nodes $u$ and $v$ in the subset of the nodes of $\sigma$ adjacent to nodes that do not belong to $\sigma$ (the boundary of $\sigma$ ) and that are linked by a path $P$ with no edge in common with $\sigma$, as depicted in Figure 3. Two other paths $P_{1}$ and $P_{2}$ between $u$ and $v$ form $\sigma$. Combining $P$ with $P_{1}$ and $P_{2}$ respectively, we obtain two new cycles $\sigma_{1}=P \oplus P_{1}$ and $\sigma_{2}=P \oplus P_{2}$. Since the covering defines a basis, $\sigma_{1}$ and $\sigma_{2}$ can be obtained by a linear combination of the cycles in the covering.

If we suppose the edges are ordered starting from those in $P_{1}$ then $P_{2}$, and finally the remaining edges, the vector corresponding to $\sigma$ has the form

$$
(\underbrace{1 \ldots 1}_{P_{1}} \underbrace{1 \ldots 1}_{P_{2}} 0 \ldots 0)
$$

while the other cycles in the basis have the form

$$
(\underbrace{0 \ldots 0}_{P_{1}} \underbrace{0 \ldots 0}_{P_{2}} \times \ldots \times)
$$


since we supposed $\sigma$ had no edge in common with any other cycle in the covering. Moreover, the vector corresponding to $\sigma_{1}$ has the form

$$
(\underbrace{1 \ldots 1}_{P_{1}} \underbrace{0 \ldots 0}_{P_{2}} \times \ldots \times) .
$$

It is clear from the form of those vectors that $\sigma_{1}$ cannot be obtained by a linear combination of cycles in the covering, which leads to a contradiction.

So each cycle in the basis shares at least one edge with some other cycle in the basis. The sum of the number of edges used in each cycle is thus greater than or equal to $2 m-n$, since at least $m-n$ repetitions of edge occur in this counting. This sum is also less than or equal to $(m-n+1) K$ since each cycle uses at most $K$ edges. We can conclude that

$$
2 m-n \leq(m-n+1) K,
$$

or, since $m$ is integral,

$$
m \geq n+\left\lceil\frac{n-K}{K-2}\right\rceil
$$

Since one of these two cases must occur, (5) or (6) is satisfied, thus the number of edges $m$ satisfies (4).

Theorem 2 provides a lower bound on the number of edges in a feasible solution. This bound is tight, as we will see in the proof of Theorem 6, and it is useful for showing that the problem is NP-complete, which is done in the next section. Moreover, a similar application of Theorem 1 leads to some classes of strong valid inequalities that are described in Section 5.

\section{Complexity}

In this section, we show that the recognition version of the $2 \mathrm{CNBR}$ problem is NP-complete for any fixed value of the bound $K$.

Problem 3 (R2CNBR) Let $G=(V, E)$ be a graph, $K \geq 3$ a given constant and $B \geq 0$ an integer. To each edge $e \in E$ is associated a cost $c_{e}$ and a unit length $d_{e}=1$. Does there exists a subset $F \subseteq E$ of edges such that $F_{K}$ is two-connected and $c(F) \leq B$ ?

Theorem $4 R 2 C N B R$ is NP-complete for any $K \geq 3$.

\section{Proof.}

It is easy to see that R2CNBR belongs to NP. We show that the Hamiltonian cycle problem reduces to R2CNBR, for any fixed $K \geq 3$. Let $G=(V, E)$ be a graph with $n=|V|$ nodes and $m=|E|$ edges, and suppose $V=\left\{v_{1}, \ldots, v_{n}\right\}$. The Hamiltonian cycle problem consists in determining if there exists a Hamiltonian cycle in $G$.

This problem is NP-complete and it can be transformed into R2CNBR in the following way. 

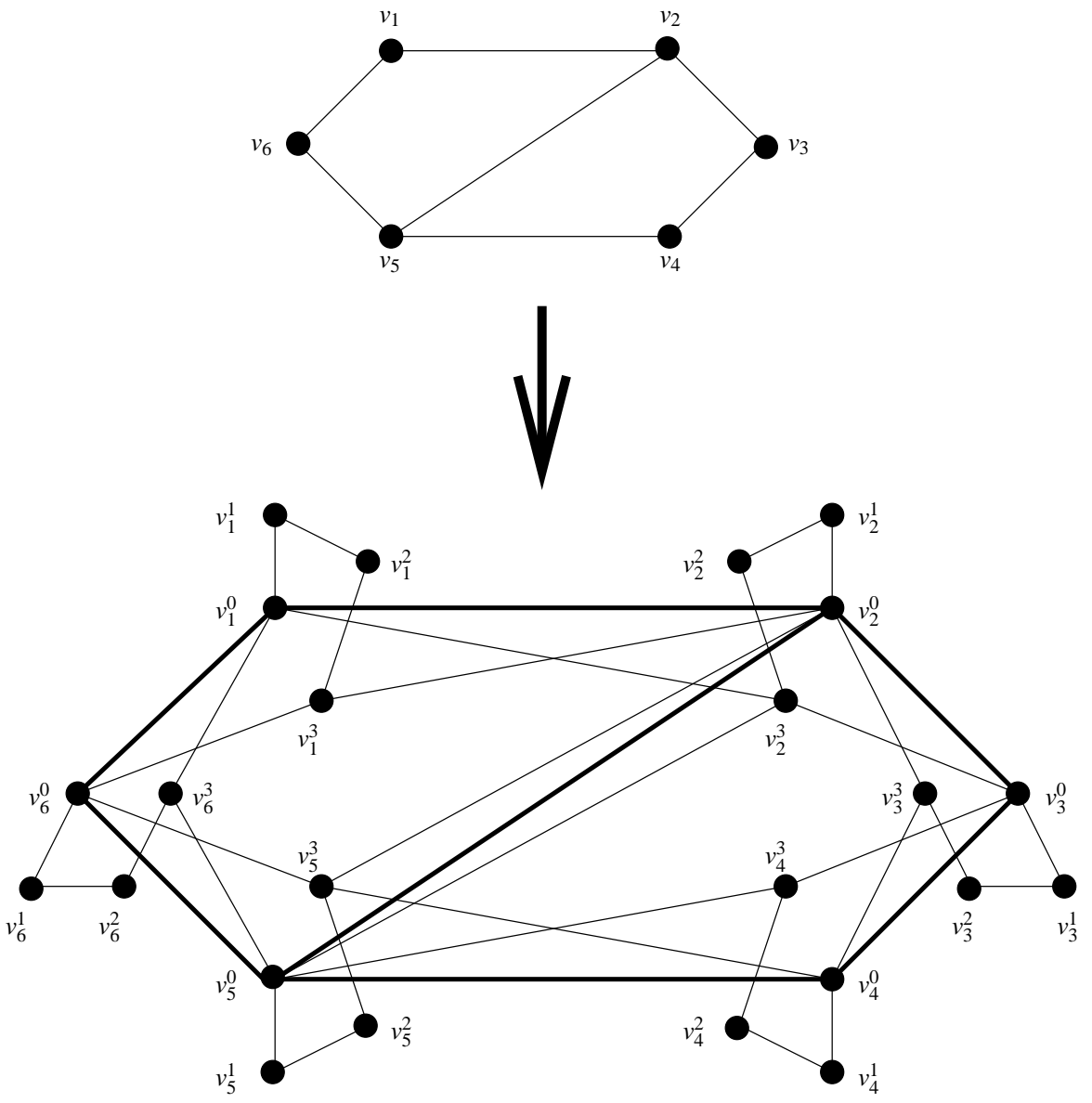

Figure 4: Transformation of Hamiltonian cycle into 2CNBR 
If $K \geq n$, finding a Hamiltonian cycle is equivalent to finding a two-connected network of cost less than or equal to $n$, for the same graph, with unit edge costs. But $K \geq n$ implies that $\mathrm{R} 2 \mathrm{CNBR}$ is equivalent to the two-connected network problem, since the constraints on the rings are redundant. Thus the Hamiltonian cycle problem reduces to R2CNBR if $K \geq n$.

If $K<n$, define a graph $G^{\prime}=\left(V^{\prime}, E^{\prime}\right)$ with

$$
V^{\prime}=\bigcup_{k=0}^{K-2} V^{k}, \text { where } V^{k}=\left\{v_{1}^{k}, \ldots, v_{n}^{k}\right\}, \quad k=0, \ldots, K-2
$$

and

$$
E^{\prime}=\bigcup_{i, j:\left\{v_{i}, v_{j}\right\} \in E}\left\{\left\{v_{i}^{0}, v_{j}^{0}\right\},\left\{v_{i}^{0}, v_{j}^{K-2}\right\},\left\{v_{j}^{0}, v_{i}^{K-2}\right\}\right\} \cup \bigcup_{k=0}^{K-3} \bigcup_{i=0}^{n}\left\{\left\{v_{i}^{k}, v_{i}^{k+1}\right\}\right\}
$$

A unit cost is again assigned to each edge. This transformation is illustrated in Figure 4 for $K=5$.

$G^{\prime}$ is composed of $n^{\prime}=(K-1) n$ nodes and $m^{\prime}=3 m+(K-2) n$ edges. The transformation can thus be performed in polynomial time and space. We will show that there exists a feasible solution of $2 \mathrm{CNBR}$ of cost less than or equal to $K n$ in $G^{\prime}$ if and only if there exists a Hamiltonian cycle in $G$, and conversely.

Suppose there exists a solution $F$ of 2 CNBR of cost less than or equal to $K n$ in $G^{\prime}$. Since edge costs are unitary, the number of edges in $F$ is equal to the cost of the solution, $c(F)$. By Theorem 2 , we thus have

$$
c(F) \geq n^{\prime}+\min \left(\left\lceil\frac{n^{\prime}-K}{K-2}\right\rceil,\left\lceil\frac{n^{\prime}}{K-1}\right\rceil\right) .
$$

But $n^{\prime}=(K-1) n$, so we have

$$
\frac{n^{\prime}}{K-1}=n
$$

and

$$
\frac{n^{\prime}-K}{K-2}=\frac{(K-1) n-K}{K-2}=n+\frac{n-K}{K-2}>n=\frac{n^{\prime}}{K-1},
$$

since $n>K$ and $K \geq 3$. Thus,

$$
K n \geq c(F) \geq n^{\prime}+\left\lceil\frac{n^{\prime}}{K-1}\right\rceil=(K-1) n+n=K n,
$$

and $F$ contains exactly $K n$ edges.

Since $F$ is two-connected, the degree of each node in this solution is at least two. Moreover, for each $v_{i}^{k}, i=1, \ldots, n, k=1, \ldots, K-3$, there are only two possible edges, and these edges must belong to any feasible solution. There are $(K-2) n$ such edges that form $n$ paths of length $K-2$, $\left(v_{i}^{0}, v_{i}^{1}, \ldots, v_{i}^{K-2}\right)$, as illustrated in bold in Figure 5. Since each edge in these paths must belong to a cycle using at most $K$ nodes, two edges $\left(v_{i}^{K-2}, w\right)$ and $\left(w, v_{i}^{0}\right)$ must be in the solution to close each path and form a feasible cycle. It is clear that the only possible choice for $w$ is a node $v_{j}^{0} \in V^{0}$.

In this way, we get a subgraph $\left(V^{\prime}, F\right)$ with $K n$ edges, with exactly $n$ edges in $F\left(V^{0}\right)$, corresponding to edges in $E$. We show that these edges form a Hamiltonian cycle in $G$. Since there are exactly $n$ edges, it is sufficient to show that these induce a two-connected subgraph of $G$, i.e. that 

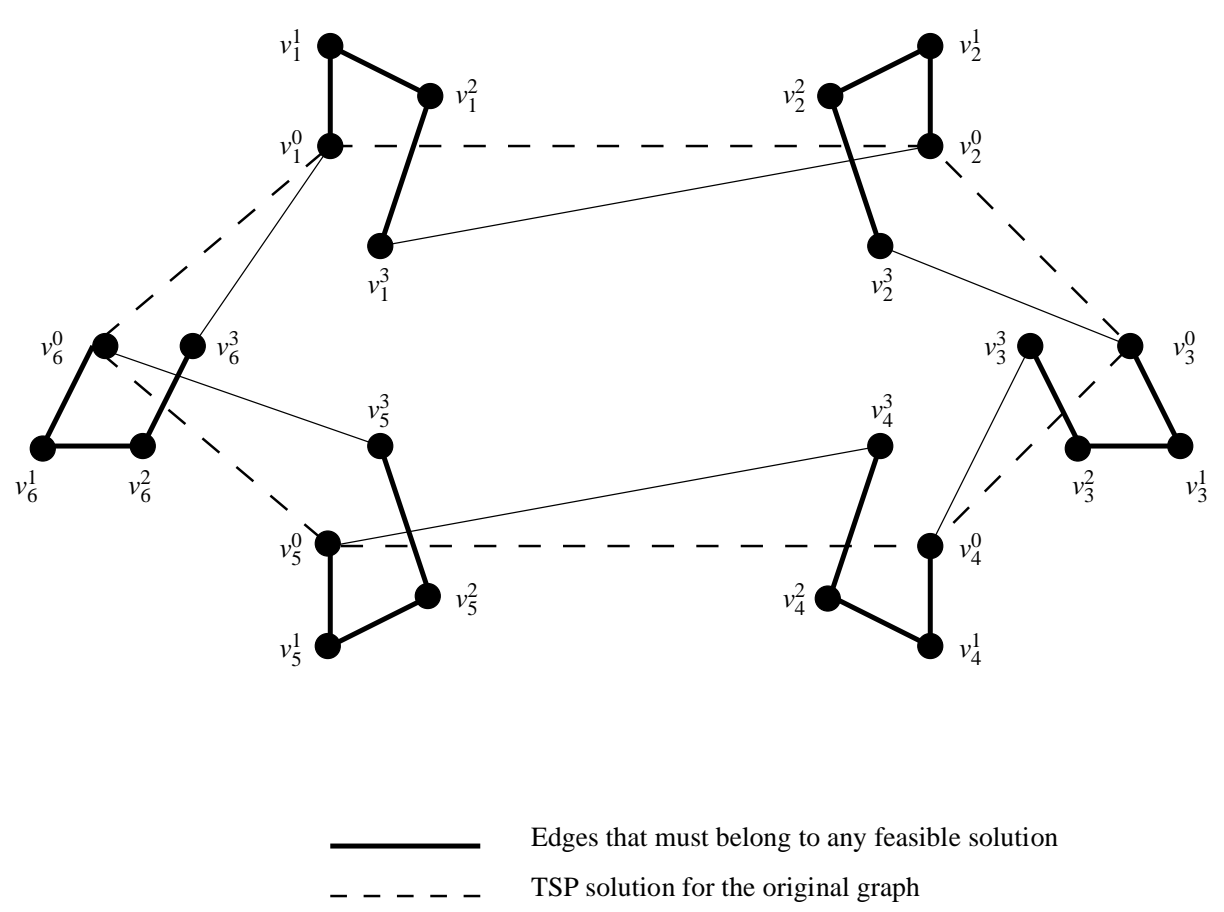

Figure 5: A solution of R2CNBR and the corresponding Hamiltonian cycle

$F\left(V^{0}\right)$ is two-connected. Let $v_{i}^{0}$ and $v_{j}^{0}$ be any two nodes in $V^{0}$. In $F$, there are two node-disjoint paths from $v_{i}^{0}$ to $v_{j}^{0}$, since $F$ is two-connected. If these paths use only nodes in $V^{0}$, the expected result holds. Otherwise, each node of these paths not belonging to $V^{0}$ must appear in a sequence of the form $v_{k}^{0}, v_{k}^{1}, \ldots, v_{k}^{K-2}, v_{l}^{0}$. By our previous construction, this imply that $\left(v_{k}^{0}, v_{l}^{0}\right)$ is also in the solution. Replacing each sequence of the form $v_{k}^{0}, v_{k}^{1}, \ldots, v_{k}^{K-2}, v_{l}^{0}$ using nodes outside $V^{0}$ by the corresponding shortcut $\left(v_{k}^{0}, v_{l}^{0}\right)$ leads to two disjoint paths from $v_{i}^{0}$ to $v_{j}^{0}$ in $E\left(V^{0}\right)$.

So, $F\left(V^{0}\right)$ is two-connected and the $n$ edges in $F\left(V^{0}\right)$ correspond to a Hamiltonian cycle in $G$.

Conversely, it is easy to see that we can complete an edge set $F\left(V^{0}\right)$ corresponding to a Hamiltonian cycle in $G$, defined by the sequence of nodes $v_{i_{1}}, v_{i_{2}}, \ldots, v_{i_{n}}, v_{i_{n+1}}=v_{i_{1}}$, into a feasible solution $F$ of 2CNBR with $K n$ edges by adding the paths $v_{i_{k}}^{0}, v_{i_{k}}^{1}, \ldots, v_{i_{k}}^{K-2}, v_{i_{k+1}}^{0}$ for $k=1, \ldots, n$ to the edges already in $F\left(V^{0}\right)$.

This concludes the proof of Theorem 4 .

\section{Cyclomatic inequalities}

From the lower bound on the number of edges in a feasible solution obtained in Section 3, we can conclude that

$$
\sum_{e \in E} x_{e} \geq M(n, K)=n+\min \left(\left\lceil\frac{n-K}{K-2}\right\rceil,\left\lceil\frac{n}{K-1}\right\rceil\right)
$$

is a valid inequality for $2 \mathrm{CNBR}$. In this section, we extend this result to new classes of valid inequalities for $2 \mathrm{CNBR}$. 


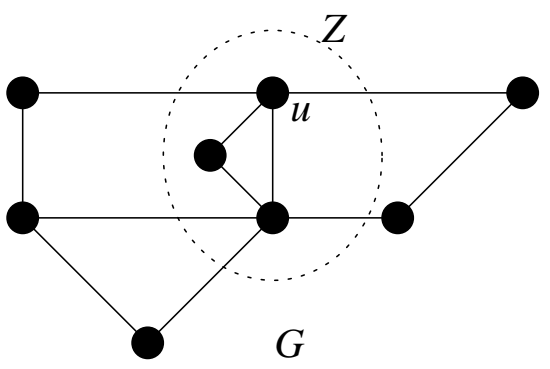

(a)

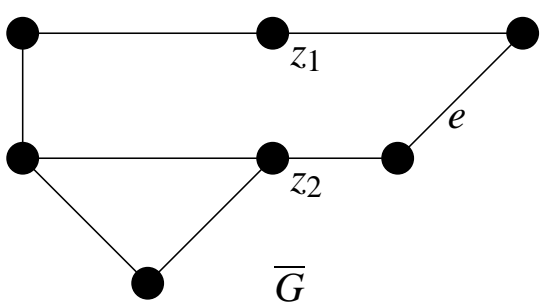

(c)

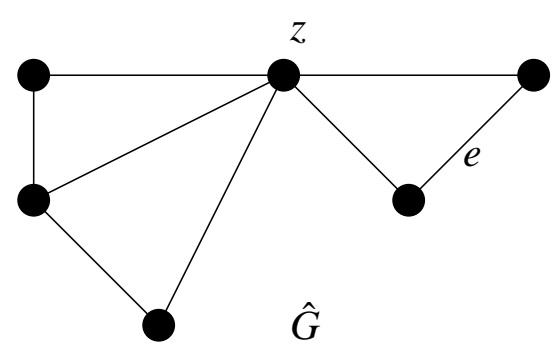

(b)

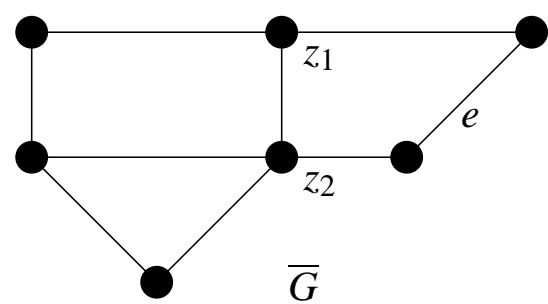

(d)

Figure 6: A graph $G$, the contracted graph $\hat{G}$ and the two steps of $\bar{G}$ construction, with a bound $K=4$.

Our first result is an extension of Theorem 2 to a partition of $V$.

Proposition 5 Let $G=(V, E)$ be a graph with $n=|V|$ nodes, $K \geq 3$ a given constant, and $W_{1}, W_{2}, \ldots, W_{p}(p \geq$ 2) a partition of $V$. Then

$$
\frac{1}{2} \sum_{i=1}^{p} x\left(\delta\left(W_{i}\right)\right) \geq M(p, K)
$$

is a valid inequality for $2 C N B R$.

Proof. Let $F$ be a feasible solution to the 2CNBR problem and let $\hat{G}$ denote the contracted graph $(V, F) / W_{1} / \ldots / W_{p}$, and $\hat{m}$ the number of edges in $\hat{G}$. We show that $\hat{m} \geq M(p, K)$.

It is easy to see that $\hat{G}$ is two-edge-connected and that each edge in $\hat{G}$ belongs to a cycle using at most $K$ edges.

If $\hat{G}$ is two-connected, Theorem 2 holds for $\hat{G}$, i.e $\hat{m} \geq M(p, k)$. Otherwise, $\hat{G}$ contains $q \geq 1$ articulation points. Let $z$ be one of these nodes, obtained after the contraction of a subset $Z \subseteq V$ of nodes in $G$ (Figure 6(a,b)). Since $F$ is two-connected, the boundary of $Z$ contains at least two nodes. Let $u$ be one of these nodes. Replacing $z$ by two nodes $z_{1}$ and $z_{2}$, and replacing each edge incident to $z$ by an edge connected to $z_{1}$ if the corresponding edge in $G$ was connected to $u$, and by an edge connected to $z_{2}$ otherwise, we obtain a graph with one articulation point less, as illustrated in Figure 6(c). However, it is possible that this new graph contains some edges not belonging to a cycle using at most $K$ edges. In this case, let $e$ be one of these edges. Before replacing $z, e$ belonged to at least one feasible cycle. Each of these cycles has been replaced by a path from $z_{1}$ 


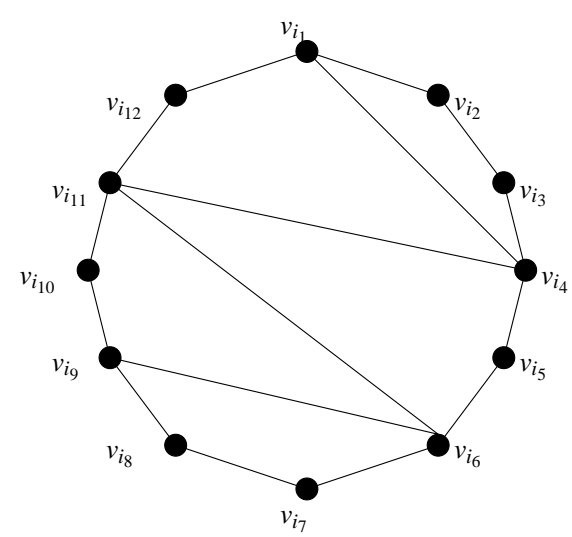

$F_{1}$

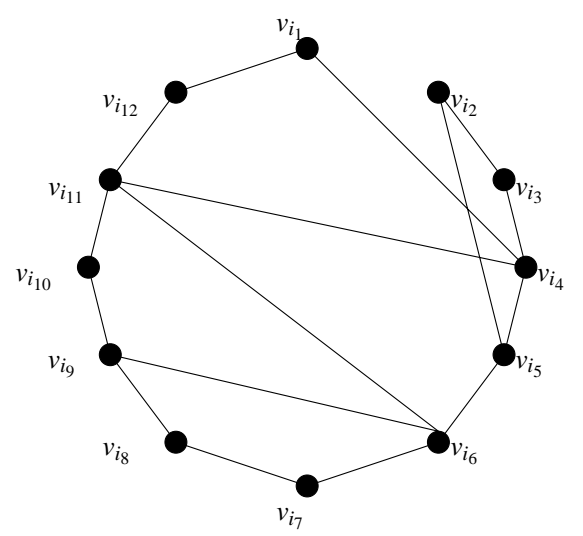

$F_{2}$

Figure 7: $F_{1}$ and $F_{2}$ for $n=12$ and $K=4$

to $z_{2}$. It is easy to see that one of these paths must contain at most $K-1$ edges, otherwise the corresponding edge in $F$ cannot belong to a feasible cycle. Therefore, adding an edge linking $z_{1}$ and $z_{2}$ is sufficient to create a feasible cycle containing $e$ (Figure 6(d)). Repeating this construction for each articulation point, we obtain a two-connected graph $\bar{G}$ such that each edge in $\bar{G}$ belongs to a cycle using at most $K$ edges. Since $\bar{G}$ has $p+q$ nodes, it contains at least $M(p+q, K)$ edges by Theorem 2 , and by our construction, it contains at most $\hat{m}+q$ edges. Therefore,

$$
\hat{m} \geq M(p+q, K)-q=p+\min \left(\left\lceil\frac{p+q-K}{K-2}\right\rceil,\left\lceil\frac{p+q}{K-1}\right\rceil\right) \geq M(p, K)
$$

and we have proved Proposition 5.

Inequalities (7) are called cyclomatic inequalities. The next theorem shows that the inequality bounding the total number of edges (i.e. $p=n$ ) is facet-defining for complete graphs.

Theorem 6 Let $G=(V, E)$ be a complete graph with $V=\left\{v_{1}, \ldots, v_{n}\right\}$, and $3 \leq K<n$ a given constant. Then $x(E) \geq M(n, K)$ defines a facet of $\mathcal{P}_{G, K}$.

Proof. We consider separately the two cases corresponding to the two possible values of $M(n, K)$.

$\mathbf{M}(\mathbf{n}, \mathbf{K})=\mathbf{n}+\left\lceil\frac{\mathbf{n}-\mathbf{K}}{\mathbf{K}-\mathbf{2}}\right\rceil$.

Let $b^{T} x \geq \beta$ be a facet-defining inequality such that the face induced by $x(E) \geq M(n, k)$ in $\mathcal{P}_{G, K}$ is contained in the facet $F_{b}$ induced by $b^{T} x \geq \beta$. Our aim is to show that $b_{e}$ has the same value for all $e \in E$.

Consider a permutation $\left\{i_{1}, \ldots, i_{n}\right\}$ of $\{1, \ldots, n\}$, and the two following sets of edges :

$$
F_{1}=\bigcup_{j=1}^{n-1}\left\{\left\{v_{i_{j}}, v_{i_{j+1}}\right\}\right\} \cup\left\{\left\{v_{i_{1}}, v_{i_{n}}\right\}\right\} \cup\left\{\left\{v_{i_{1}}, v_{i_{K}}\right\}\right\}
$$




$$
\begin{aligned}
& \cup \bigcup_{j=1}^{\left\lceil\frac{n-2}{2(K-2)}\right]-1}\left\{\left\{v_{i_{K+(j-1)(K-2)}}, v_{i_{n+1-j(K-2)}}\right\}\right\} \\
& \cup \bigcup_{j=1}^{\left\lceil\frac{n-K}{2(K-2)}\right]-1}\left\{\left\{v_{i_{n+1-j(K-2)}}, v_{i_{K+j(K-2)}}\right\}\right\}, \\
F_{2}= & \bigcup_{j=2}^{n-1}\left\{\left\{v_{i_{j}}, v_{i_{j+1}}\right\}\right\} \cup\left\{\left\{v_{i_{1}}, v_{i_{n}}\right\}\right\} \\
& \cup \bigcup_{j=1}^{\left\lceil\frac{n-2}{2(K-2)}\right\rceil-1}\left\{\left\{v_{i_{K+(j-1)(K-2)}}, v_{i_{n+1-j(K-2)}}\right\}\right\} \\
& \cup \bigcup_{j=1}^{\left\lceil\frac{n-K}{2(K-2)}\right]-1}\left\{\left\{v_{i_{n+1-j(K-2)}}, v_{i_{K+j(K-2)}}\right\}\right\} .
\end{aligned}
$$

These constructions are illustrated in Figure 7 for $n=12$ and $K=4$. It is easy to see that $F_{1}$ and $F_{2}$ define feasible solutions of $2 \mathrm{CNBR}$, and that $\left|F_{1}\right|=\left|F_{2}\right|=M(n, K)$. Therefore, the incidence vectors of $F_{1}$ and $F_{2}$ lie in $F_{b}$. Since $F_{2}$ is obtained from $F_{1}$ by replacing edge $\left\{v_{i_{1}}, v_{i_{2}}\right\}$ by $\left\{v_{i_{2}}, v_{i_{K+1}}\right\}$, we obtain that $b_{\left\{v_{i_{1}}, v_{i_{2}}\right\}}=b_{\left\{v_{i_{2}}, v_{i_{K+1}}\right\}}$.

Consider three distinct nodes $v_{i}, v_{j}$ and $v_{k}$. Since the permutation defined above was arbitrary, any permutation such that $i_{1}=i, i_{2}=j$ and $i_{K+1}=k$ leads to $b_{\left\{v_{i}, v_{j}\right\}}=b_{\left\{v_{j}, v_{k}\right\}}$. If $v_{i}, v_{j}, v_{k}$ and $v_{l}$ are four distinct nodes, we obtain by transitivity that $b_{\left\{v_{i}, v_{j}\right\}}=b_{\left\{v_{j}, v_{k}\right\}}=$ $b_{\left\{v_{k}, v_{l}\right\}}$, and therefore $b_{e}$ has the same value for all $e \in E$.

$$
\mathbf{M}(\mathbf{n}, \mathbf{K})=\mathbf{n}+\left\lceil\frac{\mathbf{n}}{\mathbf{K}-\mathbf{1}}\right\rceil .
$$

Let $b^{T} x \geq \beta$ be a facet-defining inequality such that the face induced by $x(E) \geq M(n, k)$ in $\mathcal{P}_{G, K}$ is contained in the facet $F_{b}$ induced by $b^{T} x \geq \beta$. Our aim is to show that $b_{e}$ has the same value for all $e \in E$.

Consider a permutation $\left\{i_{1}, \ldots, i_{n}\right\}$ of $\{1, \ldots, n\}$, and the two following sets of edges :

$$
\begin{aligned}
F_{3}= & \left\{\left\{v_{i_{1}}, v_{i_{n}}\right\}\right\} \cup\left\{\left\{v_{i_{1}}, v_{i_{n-K+2}}\right\}\right\} \cup \bigcup_{j=1}^{n-1}\left\{\left\{v_{i_{j}}, v_{i_{j+1}}\right\}\right\} \\
& \cup \bigcup_{j=1}^{\left\lceil\frac{n}{K-1}\right]-1}\left\{\left\{v_{i_{1+(j-1)(K-1)}}, v_{i_{1+j(K-1)}}\right\}\right\}, \\
F_{4}= & \left\{\left\{v_{i_{2}}, v_{i_{n}}\right\}\right\} \cup\left\{\left\{v_{i_{2}}, v_{i_{n-K+2}}\right\}\right\} \cup \bigcup_{j=1}^{n-1}\left\{\left\{v_{i_{j}}, v_{i_{j+1}}\right\}\right\}
\end{aligned}
$$




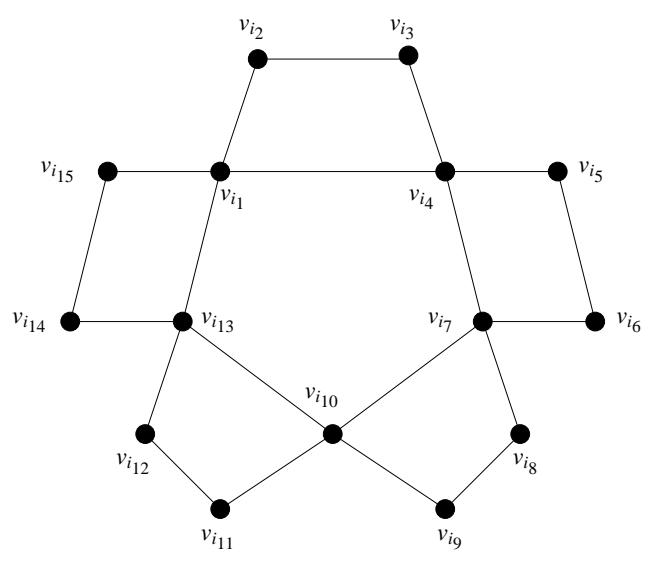

$F_{3}$

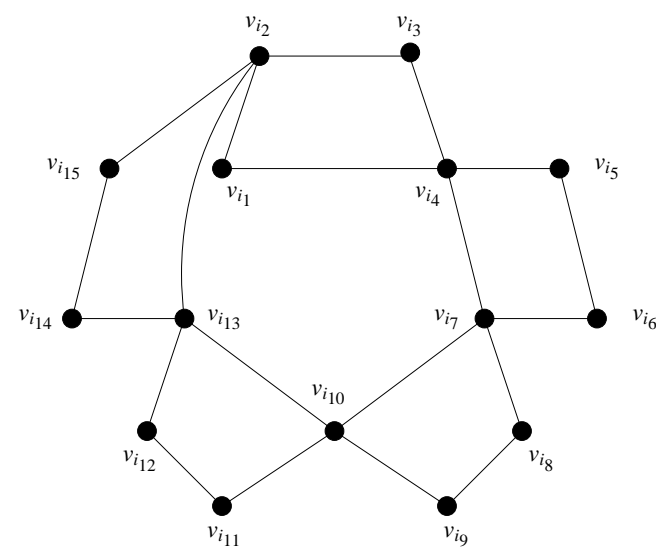

$F_{4}$

Figure 8: $F_{3}$ and $F_{4}$ for $n=15$ and $K=4$

$$
\cup \bigcup_{j=1}^{\left\lceil\frac{n}{K-1}\right\rceil-1}\left\{\left\{v_{i_{1+(j-1)(K-1)}}, v_{i_{1+j(K-1)}}\right\}\right\} .
$$

These constructions are illustrated in Figure 8 for $n=15$ and $K=4$. It is easy to see that $F_{3}$ and $F_{4}$ define feasible solutions of 2CNBR, and that $\left|F_{3}\right|=\left|F_{4}\right|=M(n, K)$. Therefore, the incidence vectors of $F_{3}$ and $F_{4}$ lie in $F_{b}$. Since $F_{4}$ is obtained from $F_{3}$ by replacing edges $\left\{v_{i_{1}}, v_{i_{n}}\right\}$ and $\left\{v_{i_{1}}, v_{i_{n-K+2}}\right\}$ by $\left\{v_{i_{2}}, v_{i_{n}}\right\}$ and $\left\{v_{i_{2}}, v_{i_{n-K+2}}\right\}$, we obtain that $b_{\left\{v_{i_{1}}, v_{i_{n}}\right\}}+$ $b_{\left\{v_{i_{1}}, v_{i_{n-K+2}}\right\}}=b_{\left\{v_{i_{2}}, v_{i_{n}}\right\}}+b_{\left\{v_{i_{2}}, v_{i_{n-K+2}}\right\}}$.

Consider four distinct nodes $v_{i}, v_{j}, v_{k}$ and $v_{l}$. Since the permutation defined above was arbitrary, any permutation such that $i_{1}=i, i_{2}=j, i_{n}=k$ and $i_{n-K+2}=l$ leads to

$$
b_{\left\{v_{i}, v_{k}\right\}}+b_{\left\{v_{i}, v_{l}\right\}}=b_{\left\{v_{j}, v_{k}\right\}}+b_{\left\{v_{j}, v_{l}\right\}} \cdot
$$

Similarly, any permutation such that $i_{1}=k, i_{2}=l, i_{n}=i$ and $i_{n-K+2}=j$ leads to

$$
b_{\left\{v_{i}, v_{k}\right\}}+b_{\left\{v_{j}, v_{k}\right\}}=b_{\left\{v_{i}, v_{l}\right\}}+b_{\left\{v_{j}, v_{l}\right\}} \text {. }
$$

This leads to $b_{\left\{v_{i}, v_{k}\right\}}=b_{\left\{v_{j}, v_{l}\right\}}$.

It remains to be shown that two edges having a common endpoint have the same coefficient. If $v_{i}, v_{j}, v_{k}, v_{l}$ and $v_{m}$ are five distinct nodes, we obtain by transitivity that $b_{\left\{v_{i}, v_{j}\right\}}=b_{\left\{v_{l}, v_{m}\right\}}=$ $b_{\left\{v_{i}, v_{k}\right\}}$, and therefore $b_{e}$ has the same value for all $e \in E$.

\section{Separation of cyclomatic inequalities}

The main difficulty that appears when trying to separate cyclomatic inequalities is the fact that the right-hand-side of the inequality is not linear in the number $p$ of subsets that define the partition. 
To override this difficulty, we decided to approximate $M(p, K)$ by a linear function of the form $a(p-1)$. Therefore, we try to find a most violated inequality of the form

$$
\frac{1}{2} \sum_{i=1}^{p} \sum_{e \in \delta\left(W_{i}\right)} \frac{x_{e}}{a} \geq p-1,
$$

which can be performed in polynomial time using Barahona's algorithm [1] for partition inequalities. If such a violated inequality is found, it is sufficient to check that the cyclomatic inequality defined by the same partition is also violated.

The effectiveness of this procedure depends heavily on the choice of $a$ in order to have a good approximation of $M(p, K)$. Our choice was to take

$$
a=\frac{n K}{(n-1)(K-1)}
$$

which leads to $a(p-1)=n+\frac{n}{K-1}$ for $p=n$. Figures 9 and 10 illustrate this approximation for $K=4$ and $K=7$, respectively, and $n=50$. We can observe that the approximation is quite accurate, and gives good results in practice.

\section{Implementation of the Branch-and-Cut algorithm}

In this section, we describe some strategic choices that were made in the implementation of our Branch-and-Cut algorithm for the 2CNBR problem. Our aim here is not to describe in detail the general Branch-and-Cut framework, but to emphasize the problem-specific aspects of our algorithm. For a general introduction to Branch-and-Cut, we refer the reader to Thienel [13] or to Nemhauser and Wolsey [11] for a more complete survey on combinatorial optimization and polyhedral theory.

The algorithm was implemented in $\mathrm{C}++$, using version 2.0 of the ABACUS library (Thienel $[13,14])$, and CPLEX 4.0 as LP-solver.

The initial linear program is defined by degree constraints $x(\delta(v)) \geq 2$ for all $v \in V$ and the lower bound on the number of edges $x(E) \geq M(n, K)$.

An important issue in the effectiveness of a Branch-and-Cut algorithm is the computation of good upper bounds. Due to the effectiveness of the Tabu Search heuristic presented in [4], we perform 600 iterations of Tabu Search in parallel with the Branch-and-Cut algorithm to obtain a good upper bound.

Moreover, we try to transform each LP-solution obtained in the Branch-and-Cut to a feasible solution by rounding up to 1 all the variables with fractional value.

The pool used to store generated inequalities is the standard pool in ABACUS. We start with a pool size equal to 100 times the number of nodes in the network, and we allow this size to be increased dynamically if necessary. All the generated inequalities are put in the pool and are dynamic, i.e. they are removed from the current LP when they are not active. The separation of valid inequalities is performed as follows. We first separate inequalities from the standard pool. If all the inequalities in the pool are satisfied by the current LP-solution, we separate inequalities in the following order : 


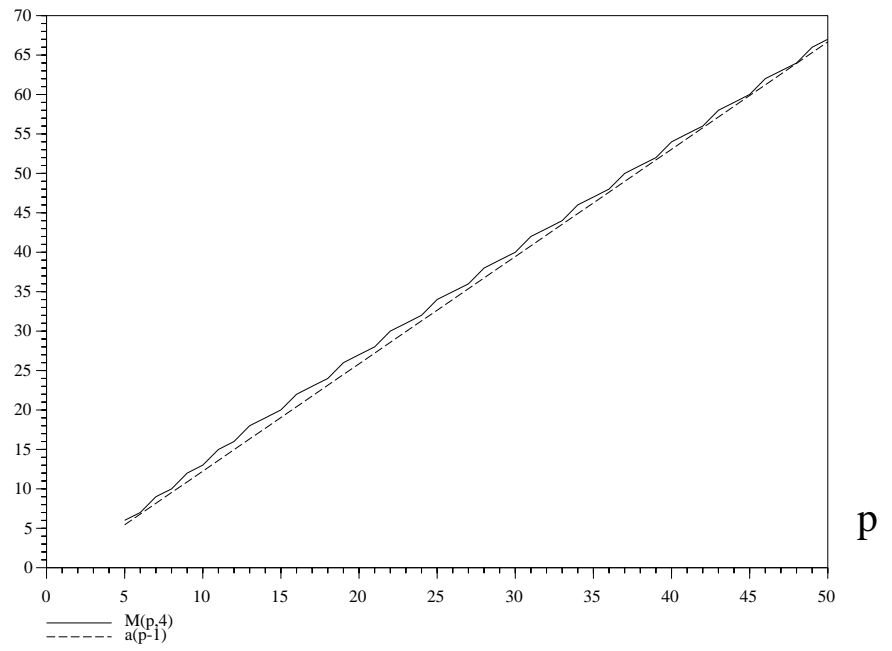

Figure 9: Approximation of $M(p, 4)$ for $n=50$

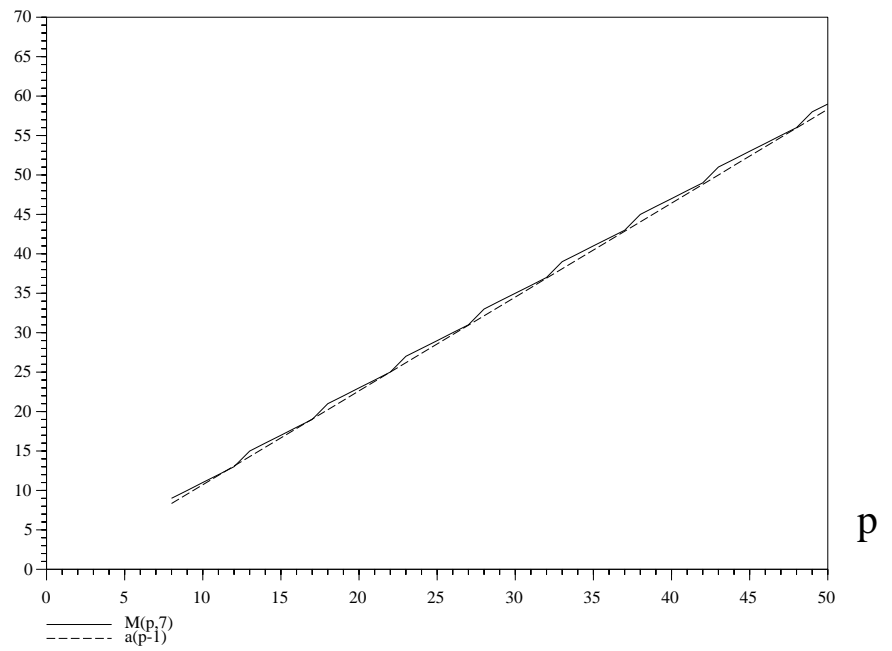

Figure 10: Approximation of $M(p, 7)$ for $n=50$ 
1. cyclomatic inequalities;

2. subset inequalities;

3. cut constraints;

4. ring-cut inequalities;

5. node-partition inequalities;

6. metric inequalities.

Subset, cut, ring-cut, node-partition and metric inequalities are studied in [5]. The order of separation was chosen after a series of numerical experiments, the choice of inequalities separated first seemingly being the best trade-off between separation time and efficiency of the cuts.

Moreover, we go to the next class of inequalities only if the number of generated cutting planes is less than 50. Otherwise, we solve the LP again and restart the separation procedure.

All inequalities are global (i.e. valid in all the tree), except ring-cut inequalities that are valid locally.

\section{Computational Results}

We present in this section numerical results obtained for the 2CNBR problem with the Branchand-Cut algorithm.

The Branch-and-Cut was implemented using ABACUS 2.0 and CPLEX 4.0, and tested on a SUN Sparc Ultra 1 workstation with a $166 \mathrm{Mhz}$ processor and 128 Mo RAM. We fixed the maximum CPU time to 10 hours, except for randomly generated problems with 40 and 50 nodes, where it was limited to 3 hours, due to the large number of problems to solve. Moreover, for these large problems, we noticed that the bounds did not improve much after 3 hours.

We consider costs equal to the rounded Euclidean distance. Tests were made for different values of the bound, for instances coming from real applications, with 12, 17, 30 and 52 nodes, and for random problems with nodes uniformly generated in a square of size $250 \times$ 250. Random problems with 10 to 50 nodes were generated, and we tested five instances of each size. Data on the randomly generated test problems are available at the Web page http: //smg.ulb.ac.be/ bfortz/2cnbm/data.html. The CPU times reported do not include the Tabu Search procedure, as it was run in parallel on another processor.

Table 2 reports results obtained for problems coming from real applications, while Table 4 reports the average results obtained for randomly generated problems. Abbreviations used in the tables are summarized in Table 1.

For 20 nodes or less, all problems could be solved to optimality. For larger problems, we remark that the problems with a small value of $K$ are much harder, the lower bound at the root of the Branch-and-Bound tree being far from the optimum.

Problems with a large value of $K$ are easier to solve due to the fact that these problems are closer to the two-connected network problem (without ring constraints), which can be solved efficiently using cut and node-partition inequalities (for the instances we considered). The most difficult cases seem to be for $K$ between 3 and 5, and especially for $K=4$. 


$\begin{array}{ll}|V| & \begin{array}{l}\text { number of nodes in the graph } \\ \text { number of edges after preprocessing } \\ \text { bound on the length of cycles }\end{array} \\ K & \begin{array}{l}\text { for random problems, total number of problems/number of problems } \\ \text { solved to optimality } \\ \text { p/o }\end{array} \\ \text { \# ineq. } & \begin{array}{l}\text { number of inequalities generated } \\ \text { node) }\end{array} \\ \text { \# B\&B noder of Branch-and-Bound nodes examined (including the root } \\ \text { LB (root) } & \begin{array}{l}\text { lower bound obtained at the root node of the Branch-and-Bound tree } \\ \text { global lower bound at the end of the optimization }\end{array} \\ \text { LB (final) } & \text { best upper bound found } \\ \text { UB } & \text { gap between the final upper and lower bounds : } \\ \text { Gap } & \text { time spent in the Branch-and-Cut (without the Tabu Search) }\end{array}$

Table 1: List of abbreviations

These instances were not tested in [6] and [5]. Our main new contribution is the introduction of cyclomatic inequalities. To test their impact on the efficiency of our algorithm, we tested the small instances coming from real applications (12 and 17 nodes) without the cyclomatic inequalities. The maximum CPU time was set to 30 minutes since all the instances were solved to optimality within this time limit using the cyclomatic inequalities. Results are reported in Table 3 and clearly show that the use of cyclomatic inequalities considerably improves the lower bounds and decreases the computing times.

\section{Conclusion}

In this paper, we study the particular case of the 2CNBR problem in which each edge has a unit length. This problem is harder to solve than its weighted counterpart. We present here new structural properties of this problem, leading to new facet-defining inequalities. Numerical results obtained with a branch-and-cut algorithm using these new inequalities as well as inequalities introduced in Fortz et al. [6] and Fortz and Labbé [5] are reported. From these results, we can conclude that the Branch-and-Cut algorithm is able to solve to optimality instances of small size (up to 30 nodes). However, problems with a small value of the bound (3 to 5) remain difficult to deal with and should probably need specific methods. 


\begin{tabular}{ccccccccccc}
\hline$|V|$ & $K$ & $|E|$ & \# ineq. \# B\&B & LB & LB & UB & Gap & Gap & CPU time \\
nodes & (root) & (final) & (root) & (final) & (hh:mm:ss) \\
\hline 12 & 39 & 3 & 179 & 31 & 740 & 794 & 794 & 7.3 & 0.0 & $0: 00: 09$ \\
12 & 39 & 4 & 288 & 105 & 647 & 681 & 681 & 5.3 & 0.0 & $0: 00: 18$ \\
12 & 39 & 5 & 73 & 7 & 598 & 606 & 606 & 1.3 & 0.0 & $0: 00: 01$ \\
12 & 39 & 6 & 53 & 5 & 563 & 568 & 568 & 0.9 & 0.0 & $0: 00: 01$ \\
12 & 39 & 8 & 39 & 13 & 521 & 537 & 537 & 3.1 & 0.0 & $0: 00: 01$ \\
12 & 39 & 10 & 27 & 7 & 517 & 521 & 521 & 0.8 & 0.0 & $0: 00: 01$ \\
12 & 39 & 16 & 24 & 1 & 496 & 496 & 496 & 0.0 & 0.0 & $0: 00: 01$ \\
\hline 17 & 88 & 3 & 1570 & 3521 & 1034 & 1100 & 1100 & 6.4 & 0.0 & $0: 18: 29$ \\
17 & 88 & 4 & 2549 & 5381 & 907 & 966 & 966 & 6.5 & 0.0 & $0: 29: 37$ \\
17 & 88 & 5 & 270 & 61 & 837 & 855 & 855 & 2.2 & 0.0 & $0: 00: 26$ \\
17 & 88 & 6 & 75 & 1 & 797 & 797 & 797 & 0.0 & 0.0 & $0: 00: 02$ \\
17 & 88 & 8 & 222 & 105 & 752 & 766 & 766 & 1.9 & 0.0 & $0: 00: 22$ \\
17 & 88 & 10 & 36 & 1 & 715 & 715 & 715 & 0.0 & 0.0 & $0: 00: 01$ \\
17 & 88 & 16 & 13 & 1 & 711 & 711 & 711 & 0.0 & 0.0 & $0: 00: 01$ \\
\hline 30 & 200 & 3 & 8481 & 38011 & 1310 & 1417 & 1431 & 9.2 & 1.0 & $10: 00: 00$ \\
30 & 200 & 4 & 23545 & 33887 & 1171 & 1227 & 1326 & 13.2 & 8.1 & $10: 00: 00$ \\
30 & 200 & 5 & 22860 & 35693 & 1078 & 1127 & 1176 & 9.1 & 4.3 & $10: 00: 00$ \\
30 & 200 & 6 & 2683 & 2721 & 1018 & 1055 & 1055 & 3.6 & 0.0 & $0: 45: 22$ \\
30 & 200 & 8 & 1957 & 1049 & 930 & 956 & 956 & 2.8 & 0.0 & $0: 18: 21$ \\
30 & 200 & 10 & 315 & 9 & 895 & 901 & 901 & 0.7 & 0.0 & $0: 00: 41$ \\
30 & 200 & 16 & 1650 & 781 & 843 & 861 & 861 & 2.1 & 0.0 & $0: 08: 31$ \\
\hline 52 & 622 & 3 & 4143 & 11115 & 1630 & 1674 & 1870 & 14.7 & 11.7 & $10: 00: 00$ \\
52 & 622 & 4 & 5129 & 5783 & 1411 & 1457 & 1663 & 17.9 & 14.1 & $10: 00: 00$ \\
52 & 622 & 5 & 6474 & 3781 & 1303 & 1354 & 1478 & 13.4 & 9.2 & $10: 00: 00$ \\
52 & 622 & 6 & 5233 & 4099 & 1256 & 1292 & 1362 & 8.4 & 5.4 & $10: 00: 00$ \\
52 & 622 & 8 & 5974 & 3049 & 1178 & 1209 & 1246 & 5.8 & 3.1 & $10: 00: 00$ \\
52 & 622 & 10 & 7535 & 3933 & 1136 & 1160 & 1208 & 6.3 & 4.1 & $10: 00: 00$ \\
52 & 622 & 16 & 10117 & 4693 & 1074 & 1091 & 1104 & 2.8 & 1.2 & $10: 00: 00$ \\
\hline & & & & & & & & & &
\end{tabular}

Table 2: Branch-and-Cut results, real applications, unit edge lengths 


\begin{tabular}{cccccccccccc}
\hline$|V|$ & $K$ & $|E|$ \# ineq. \# B\&B & LB & LB & UB & Gap & Gap & CPU time \\
nodes (root) & (final) & \multicolumn{3}{c}{ (root) } & (final) & $($ hh:mm:ss) \\
\hline 12 & 39 & 3 & 566 & 371 & 689 & 794 & 794 & 15.2 & 0.0 & $0: 01: 14$ \\
12 & 39 & 4 & 1203 & 1311 & 606 & 681 & 681 & 12.4 & 0.0 & $0: 02: 54$ \\
12 & 39 & 5 & 542 & 265 & 565 & 606 & 606 & 7.3 & 0.0 & $0: 00: 30$ \\
12 & 39 & 6 & 221 & 67 & 544 & 568 & 568 & 4.4 & 0.0 & $0: 00: 08$ \\
12 & 39 & 8 & 105 & 33 & 513 & 537 & 537 & 4.7 & 0.0 & $0: 00: 02$ \\
12 & 39 & 10 & 94 & 19 & 501 & 521 & 521 & 4.0 & 0.0 & $0: 00: 01$ \\
12 & 39 & 16 & 24 & 1 & 496 & 496 & 496 & 0.0 & 0.0 & $0: 00: 01$ \\
\hline 17 & 88 & 3 & 2956 & 5449 & 965 & 1057 & 1108 & 14.8 & 4.8 & $0: 30: 00$ \\
17 & 88 & 4 & 6015 & 5135 & 853 & 911 & 966 & 13.2 & 6.0 & $0: 30: 00$ \\
17 & 88 & 5 & 1960 & 1773 & 798 & 855 & 855 & 7.1 & 0.0 & $0: 08: 52$ \\
17 & 88 & 6 & 784 & 189 & 772 & 797 & 797 & 3.2 & 0.0 & $0: 01: 17$ \\
17 & 88 & 8 & 1134 & 567 & 731 & 766 & 766 & 4.8 & 0.0 & $0: 02: 19$ \\
17 & 88 & 10 & 71 & 9 & 709 & 715 & 715 & 0.8 & 0.0 & $0: 00: 02$ \\
17 & 88 & 16 & 217 & 113 & 693 & 711 & 711 & 2.6 & 0.0 & $0: 00: 13$ \\
\hline
\end{tabular}

Table 3: Branch-and-Cut results for unit edge lengths without cyclomatic inequalities 


\begin{tabular}{ccccccccc}
\hline$|V|$ & $|E|$ & $K$ & p/o & \# ineq. & \# B\&B & $\begin{array}{c}\text { Gap } \\
\text { nodes }\end{array}$ & $\begin{array}{c}\text { Gap } \\
\text { (root) }\end{array}$ (final) & $\begin{array}{c}\text { CPU } \\
\text { (hh:mm:ss) }\end{array}$ \\
\hline 10 & 25.6 & 3 & $5 / 5$ & 177.8 & 64.2 & 5.7 & 0.0 & $0: 00: 08$ \\
10 & 25.6 & 4 & $5 / 5$ & 93.6 & 35.0 & 4.7 & 0.0 & $0: 00: 03$ \\
10 & 25.6 & 5 & $5 / 5$ & 72.0 & 20.6 & 2.5 & 0.0 & $0: 00: 02$ \\
10 & 25.6 & 6 & $5 / 5$ & 48.8 & 14.6 & 3.6 & 0.0 & $0: 00: 01$ \\
10 & 25.6 & 8 & $5 / 5$ & 17.0 & 5.4 & 1.0 & 0.0 & $0: 00: 01$ \\
10 & 25.6 & 10 & $5 / 5$ & 3.2 & 1.0 & 0.0 & 0.0 & $0: 00: 01$ \\
\hline 20 & 74.4 & 3 & $5 / 4$ & 2763.8 & 17192.2 & 8.0 & 1.1 & $2: 15: 54$ \\
20 & 68.7 & 4 & $5 / 5$ & 6454.4 & 24128.2 & 8.4 & 0.0 & $2: 29: 58$ \\
20 & 68.7 & 5 & $5 / 5$ & 582.8 & 477.4 & 4.4 & 0.0 & $0: 02: 31$ \\
20 & 68.7 & 6 & $5 / 5$ & 355.0 & 169.8 & 3.7 & 0.0 & $0: 00: 58$ \\
20 & 68.7 & 8 & $5 / 5$ & 322.8 & 169.4 & 3.4 & 0.0 & $0: 00: 42$ \\
20 & 68.7 & 10 & $5 / 5$ & 228.8 & 37.8 & 2.1 & 0.0 & $0: 00: 19$ \\
20 & 68.7 & 16 & $5 / 5$ & 68.6 & 7.0 & 0.7 & 0.0 & $0: 00: 01$ \\
\hline 30 & 179.4 & 3 & $5 / 2$ & 4845.6 & 26101.4 & 10.8 & 3.9 & $7: 54: 33$ \\
30 & 179.4 & 4 & $5 / 1$ & 15326.2 & 26165.4 & 12.2 & 6.3 & $8: 46: 06$ \\
30 & 179.4 & 5 & $5 / 1$ & 15310.0 & 21576.6 & 11.1 & 5.3 & $8: 03: 46$ \\
30 & 179.4 & 6 & $5 / 4$ & 7763.2 & 13739.8 & 6.4 & 1.0 & $5: 03: 45$ \\
30 & 179.4 & 8 & $5 / 5$ & 2471.2 & 2261.4 & 4.1 & 0.0 & $0: 55: 43$ \\
30 & 179.4 & 10 & $5 / 5$ & 2652.8 & 1824.6 & 3.1 & 0.0 & $0: 43: 14$ \\
30 & 179.4 & 16 & $5 / 5$ & 2264.0 & 705.4 & 2.7 & 0.0 & $0: 16: 27$ \\
\hline 40 & 231.6 & 3 & $5 / 0$ & 2237.4 & 8677.0 & 10.7 & 5.8 & $3: 00: 00$ \\
40 & 231.6 & 4 & $5 / 0$ & 3790.6 & 7089.0 & 10.9 & 7.0 & $3: 00: 00$ \\
40 & 231.6 & 5 & $5 / 0$ & 4203.8 & 6433.8 & 10.3 & 5.8 & $3: 00: 00$ \\
40 & 231.6 & 6 & $5 / 0$ & 4863.0 & 7096.2 & 8.6 & 4.3 & $3: 00: 00$ \\
40 & 231.6 & 8 & $5 / 2$ & 4336.4 & 4258.2 & 5.6 & 1.4 & $2: 07: 27$ \\
40 & 231.6 & 10 & $5 / 2$ & 4573.8 & 3095.0 & 5.7 & 1.6 & $2: 00: 23$ \\
40 & 231.6 & 16 & $5 / 4$ & 3850.8 & 1889.8 & 3.1 & 0.4 & $1: 14: 11$ \\
\hline 50 & 337.2 & 3 & $5 / 0$ & 2089.4 & 4400.6 & 12.8 & 9.3 & $3: 00: 00$ \\
50 & 337.2 & 4 & $5 / 0$ & 3123.6 & 3736.2 & 15.9 & 12.2 & $3: 00: 00$ \\
50 & 337.2 & 5 & $5 / 0$ & 3680.6 & 3312.2 & 13.3 & 9.7 & $3: 00: 00$ \\
50 & 337.2 & 6 & $5 / 0$ & 3871.6 & 3417.4 & 12.3 & 8.9 & $3: 00: 00$ \\
50 & 337.2 & 8 & $5 / 0$ & 4227.4 & 2871.4 & 8.1 & 4.9 & $3: 00: 00$ \\
50 & 337.2 & 10 & $5 / 0$ & 4315.8 & 2744.2 & 5.8 & 2.8 & $3: 00: 00$ \\
50 & 337.2 & 16 & $5 / 1$ & 4448.4 & 2290.2 & 3.5 & 1.3 & $2: 28: 00$ \\
\hline & & & & & & & &
\end{tabular}

Table 4: Branch-and-Cut results, random networks, unit edge lengths 


\section{References}

[1] F. Barahona. Separating from the dominant of the spanning tree polytope. Op. Research Letters, 12:201-203, 1992.

[2] C. Berge. Graphs and hypergraphs. North-Holland, Amsterdam, 1973.

[3] S.C. Boyd and T. Hao. An integer polytope related to the design of survivable communication networks. SIAM J. Discrete Math., 6(4):612-630, 1993.

[4] B. Fortz. Design of Survivable Networks with Bounded Rings, volume 2 of Network Theory and Applications. Kluwer Academic Publishers, 2000.

[5] B. Fortz and M. Labbé. Polyhedral results for two-connected networks with bounded rings. Technical Report IAG 03/01, Université Catholique de Louvain, Institut d'Administration et de Gestion, 2001. To appear in Mathematical Programming.

[6] B. Fortz, M. Labbé, and F. Maffioli. Solving the two-connected network with bounded meshes problem. Operations Research, 48(6):866-877, 2000.

[7] M. Grötschel and C.L. Monma. Integer polyhedra arising from certain design problems with connectivity constraints. SIAM J. Discrete Math., 3:502-523, 1990.

[8] M. Grötschel, C.L. Monma, and M. Stoer. Computational results with a cutting plane algorithm for designing communication networks with low-connectivity constraints. Operations Research, 40(2):309-330, 1992.

[9] M. Grötschel, C.L. Monma, and M. Stoer. Design of Survivable Networks, volume 7 on Network models of Handbooks in OR/MS, chapter 10, pages 617-672. North-Holland, 1995.

[10] C.L. Monma and D.F. Shallcross. Methods for designing communications networks with certain two-connected survivability constraints. Operations Research, 37(4):531-541, 1989.

[11] G.L. Nemhauser and L.A. Wolsey. Integer and combinatorial optimization. WileyInterscience series in discrete mathematics and optimization. Wiley, 1988.

[12] K. Steiglitz, P. Weiner, and D.J. Kleitman. The design of minimum-cost survivable networks. IEEE Transactions on Circuit Theory, CT-16:455-460, 1969.

[13] S. Thienel. ABACUS-A Branch-And-Cut System. PhD thesis, Universität zu Köln, 1995.

[14] S. Thienel. ABACUS-A Branch-And-Cut System, Version 2.0, Users's Guide and Reference Manual. Universität zu Köln, 1997. 